\title{
PLANE STRAIN DEFORMATION TEXTURES OF $\beta-\mathrm{Si}_{3} \mathbf{N}_{4}$
}

\author{
PETER MILLER, DAVID COLLINS, INNA GOLINKIN AND KEITH BOWMAN \\ School of Materials Engineering, Purdue University, West Lafayette, IN 47907
}

(Received 2 August 1994)

Several prior investigations have documented deformation-induced rotation of $\beta-\mathrm{Si}_{3} \mathrm{~N}_{4}$ grains resulting in texture and property anisotropy. As documented previously for axisymmetric textures, Jeffery-based models correlate well with experimental results for a limited range of strains in plane strain deformation. Comparison to other experimental results and the implications of these results on texture modeling are discussed.

KEY WORDS: Silicon nitride, forging, texture, difference pole figures, superplastic forming.

\section{INTRODUCTION}

Both the mechanical and thermal properties of silicon nitride $\left(\mathrm{Si}_{3} \mathrm{~N}_{4}\right)$ depend strongly on the orientation distribution of the elongated $\beta-\mathrm{Si}_{3} \mathrm{~N}_{4}$ grains in commercial cutting tools and engine parts. Deformation of the bulk material, through methods such as axisymmetric compression, induces a preferred orientation of $\beta-\mathrm{Si}_{3} \mathrm{~N}_{4}$ grains that affects mechanical properties. In an earlier investigation on processing of $\beta$-Si ${ }_{3} \mathrm{~N}_{4}$, Lee and Bowman (1994) applied a hydrodynamic model developed by Jeffery (1922). This model describes the reorientation of non-interacting, rigid ellipsoids in an incompressible Newtonian flow. Given the shape of the particle, the applied strain and the initial orientation for each grain, this adaptation was used to calculate the final orientation distribution induced by deformation. Experimental data and model predictions for axisymmetric compression agreed favorably for height reductions up to $60 \%$. The discrepancies that occurred at larger height reductions were attributed to intergranular interactions and non-Newtonian rheology. The present study is an application of the Jeffery approach to predict texture development in $\beta-\mathrm{Si}_{3} \mathrm{~N}_{4}$ with approximately $15 \mathrm{wt} \%$ of sintering additives. These model predictions are compared to experimental results from plane strain compression. Furthermore, because the previous investigation by Lee and Bowman only considered texture maxima, complete orientations in the plane strain condition resolved by modeling and experiment were evaluated. 


\section{ROTATION OF ELONGATED GRAINS UNDER PLANE STRAIN COMPRESSION}

Jeffery's general equations of motion for an ellipsoid embedded in a viscous, incompressible Newtonian fluid assume that each grain is totally isolated within the fluid phase and that motion is large relative to particle size (Jeffery, 1922). The resulting deformation model relates to the rotation rates with the applied strain. For plane strain compression $\left(\sigma_{Z}=-\sigma_{Y}\right.$ and $\left.\sigma_{X}=0\right)$, the rotation rates for pure shear $\left(\sigma_{X}=-\sigma_{Y}\right.$ and $\left.\sigma_{Z}=0\right)$ deformation developed by Gay (1968) can be used even though the stress states are dissimilar because the predictions are strain dependent. These rotation rates are given as

$$
\begin{gathered}
\dot{\phi}=\cos \theta=0 \\
\dot{\theta}=-\frac{1}{2} \dot{\varepsilon} \frac{\left(a^{2}-b^{2}\right)}{\left(a^{2}+b^{2}\right)} \sin 2 \theta \cos 2 \phi \\
\dot{\phi}=\dot{\varepsilon} \frac{\left(a^{2}-b^{2}\right)}{\left(a^{2}+b^{2}\right)} \sin 2 \phi
\end{gathered}
$$

where $\phi$ and $\theta$ are the Euler angles describing the grain orientation illustrated in Figure 1. The axes of an axisymmetric ellipsoid are given by $a$ and $b, a$ designated as the major axis of symmetry. The terms $\dot{\theta}$ and $\dot{\phi}$ are the corresponding time derivatives of the rotation angles and $\dot{\varepsilon}$ is the effective strain rate. Integration of Eq. (4c) yields a relationship between the initial and final angles, $\theta_{i}$ and $\theta_{f}$ respectively, as a function of the deformation strain. This can be written as

$$
\ln \frac{\left(\tan \theta_{f}\right)}{\left(\tan \theta_{i}\right)}=2 \varepsilon \frac{R^{2}-1}{R^{2}+1}
$$

where $R$ is the grain aspect ratio, $a / b$, and $\varepsilon$ is the true normal compression strain. If the quotient of Eq. (4c) and Eq. (4b) is integrated, the initial and final values of $\phi$ can be related to those of $\theta$ as

$$
\frac{\tan ^{2} \theta_{f}}{\tan ^{2} \theta_{i}}=\frac{\sin ^{2} \phi_{i}}{\sin ^{2} \phi_{f}}
$$

Input of the grain aspect ratio, the strain resulting from plane strain compression and an initial orientation into Eq. (5) and Eq. (6) yields predictions of the final grain orientations. However, as stated above, these equations were developed only for dilute solutions having no particle-particle interactions.

Hosford has developed another approach for reorientation of elongated grains. This geometric method focuses on the rotation of lines drawn in a material during axisymmetric deformation (Hosford, 1993). This solution allows the lines, representing grains, to change in length without interactions, yielding an infinite texture maxima. Superplastic tensile experiments by $\mathrm{Wu}$ and Chen (1992) applied this model and in fact, saw grain lengthening due to welding, or cohesion, of silicon nitride grains during axisymmetric elongation. 


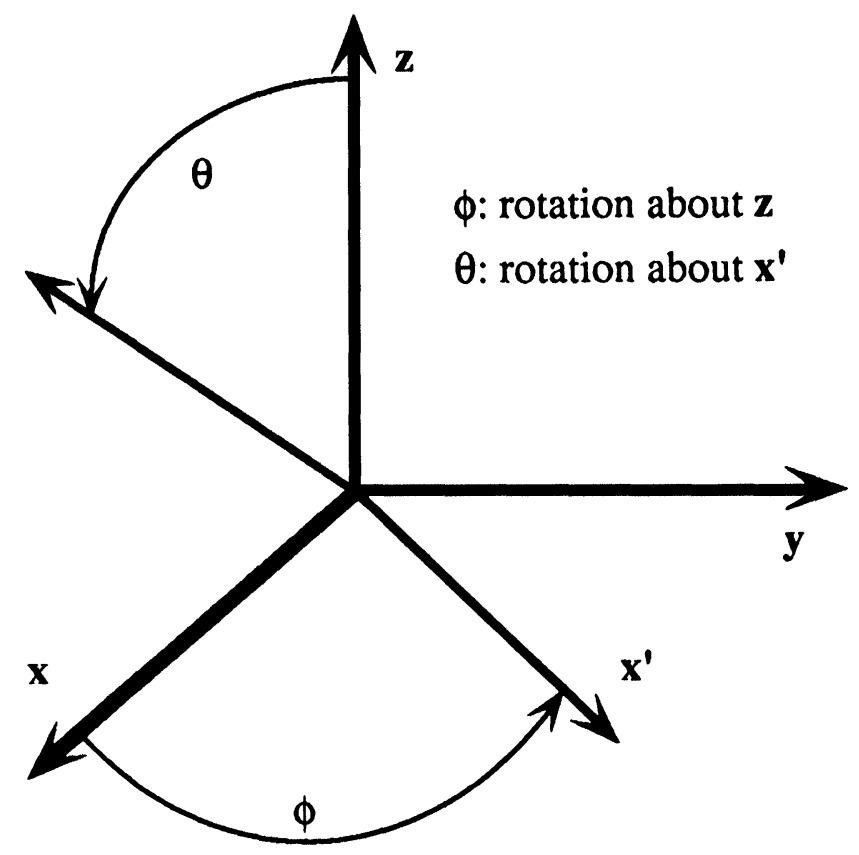

Figure 1 Euler Rotations, $\phi$ and $\theta$.

To address particle interaction in a flow field, Folgar and Tucker developed a model to predict orientation patterns of concentrated solutions of large fibers (Folgar, 1984). The model combines a hydrodynamic term which is equivalent to the Jeffery model with an infinite aspect ratio, i.e. $\left(R^{2}-1\right) /\left(R^{2}+1\right)$ equal to unity, and a fiber interaction term analogous to rotary diffusivity due to Brownian motion (Advani, 1987). This interaction term can be represented as a empirical scalar of the strain rate tensor, where the scalar is related to the aspect ratio and the concentration. Model predictions were compared to experimental orientation patterns determined from concentrated solution of 6,6-Nylon fibers with $R=16$ in silicon oil undergoing simple and pure shear, as in injection molding (Folgar, 1983). For the various concentrations investigated, up to $16 \mathrm{vol} \%$, the fit between the model and the experimental results agreed favorably when the interaction parameter was in the range between 0.01 and 0.003 (Folgar, 1983).

\section{EXPERIMENTAL PROCEDURES}

\section{Material and Specimen Preparation}

Starting powders generally consist of $\alpha-\mathrm{Si}_{3} \mathrm{~N}_{4}$ obtained through nitridation of silicon during powder synthesis. At sintering temperatures between 1650 and $2000^{\circ} \mathrm{C}$, the nearly equiaxed $\alpha-\mathrm{Si}_{3} \mathrm{~N}_{4}$ powder transforms to fibrous $\beta-\mathrm{Si}_{3} \mathrm{~N}_{4}$ (see Figure 2) (Drew, 1988; $\mathrm{Wu}$, 1992). Densification requires some sintering aids, e.g. $\mathrm{Y}_{2} \mathrm{O}_{3}$ and $\mathrm{Al}_{2} \mathrm{O}_{3}$. At high 


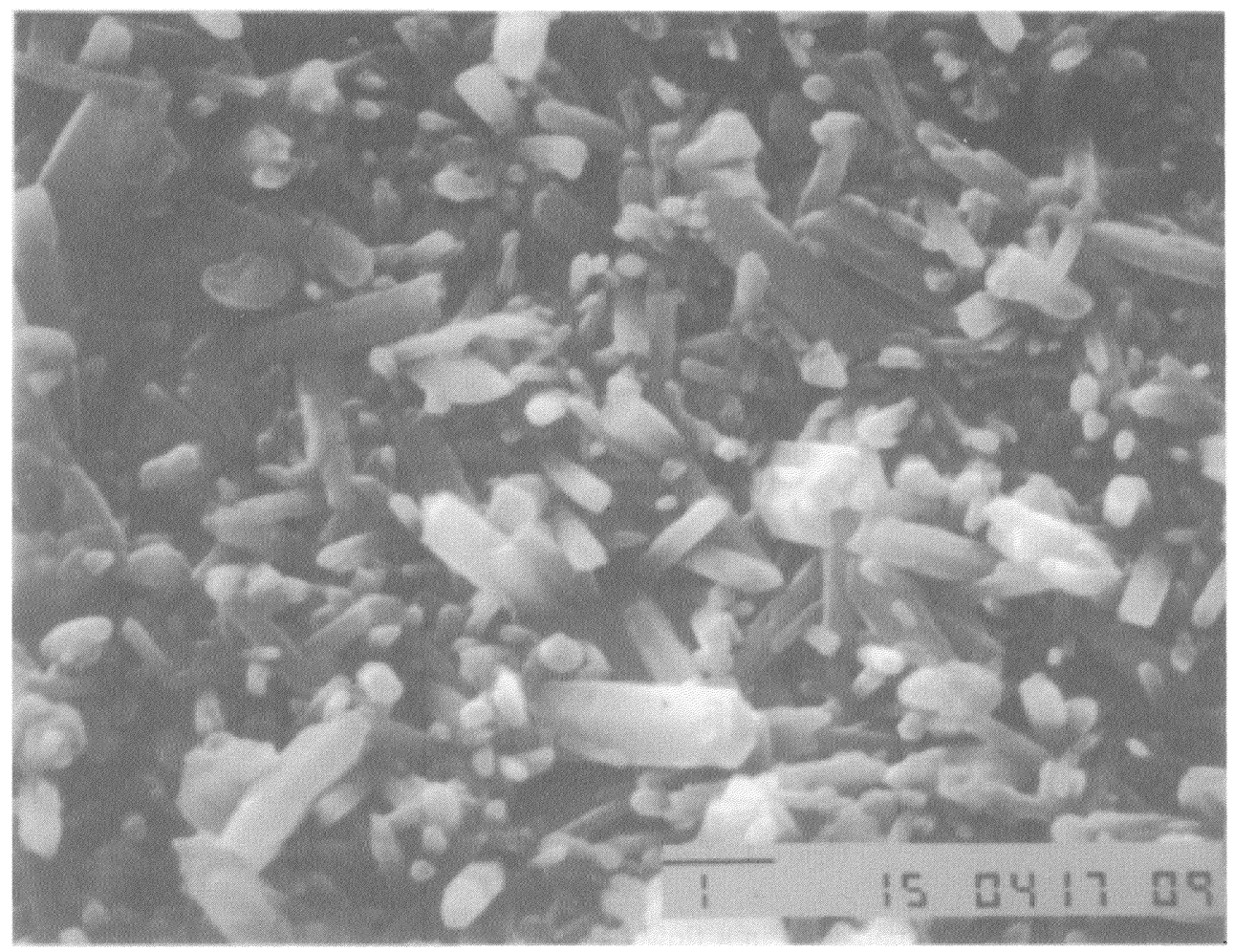

Figure 2 Elongated microstructure of $\beta-\mathrm{Si}_{3} \mathrm{~N}_{4}$ formed after densification by hot-pressing. Sample etched in $1 \mathrm{LiOH}: 2 \mathrm{NaOH}: 2 \mathrm{KOH}$ molten salt bath for three minutes at $550^{\circ} \mathrm{C}$.

temperatures, $\mathrm{Si}_{3} \mathrm{~N}_{4}$ reacts with these additives to form an oxynitride liquid that promotes liquid-phase sintering. Upon cooling, a glassy phase containing the sintering additives is usually formed at $\beta-\mathrm{Si}_{3} \mathrm{~N}_{4}$ grain boundaries.

Nearly equiaxed $\alpha$-phase $\mathrm{Si}_{3} \mathrm{~N}_{4}$ powder (H. C. Starck LC-12SX) of $0.5 \mu \mathrm{m}$ average size was ball milled with alumina media for two hours with $15 \mathrm{wt} \%$ sintering additives of yttrium-aluminum-garnet (YAG) made of the following constituents: 3 mole\% of $\mathrm{Y}_{2} \mathrm{O}_{3}$ (H. C. Starck "finest") and 5 mole\% of $\mathrm{Al}_{2} \mathrm{O}_{3}$ (Fisher A591-500). The powder was then furnace dried for 24 hours at $110^{\circ} \mathrm{C}$ and cold-pressed into $3.8 \mathrm{~cm}$ diameter billets approximately $5 \mathrm{~cm}$ high at applied pressures less than $1 \mathrm{MPa}$.

The cold-pressed green bodies were sintered at $1650^{\circ} \mathrm{C}$ in 0.5 atm of nitrogen gas. During this procedure, the nearly equiaxed $\alpha$-phase $\mathrm{Si}_{3} \mathrm{~N}_{4}$ transformed into $\beta$-phase $\mathrm{Si}_{3} \mathrm{~N}_{4}$, with resulting densities of approximately $98 \%$ theoretical. Since no pressure was applied, the resulting sample did not develop a texture and was subsequently used for defocusing corrections (see section 3.2).

Following sintering, the billets were hot-pressed at $1650^{\circ} \mathrm{C}$ in a $3.8 \mathrm{~cm}$ graphite die for 2 hours at an applied pressure of $10 \mathrm{MPa}$ in 0.5 atm of $\mathrm{N}_{2}$. The resulting samples attained $\sim 99 \%$ theoretical density (cf. Figure 2), while exhibiting only slight fiber textures from powder consolidation (Lee, 1992). 


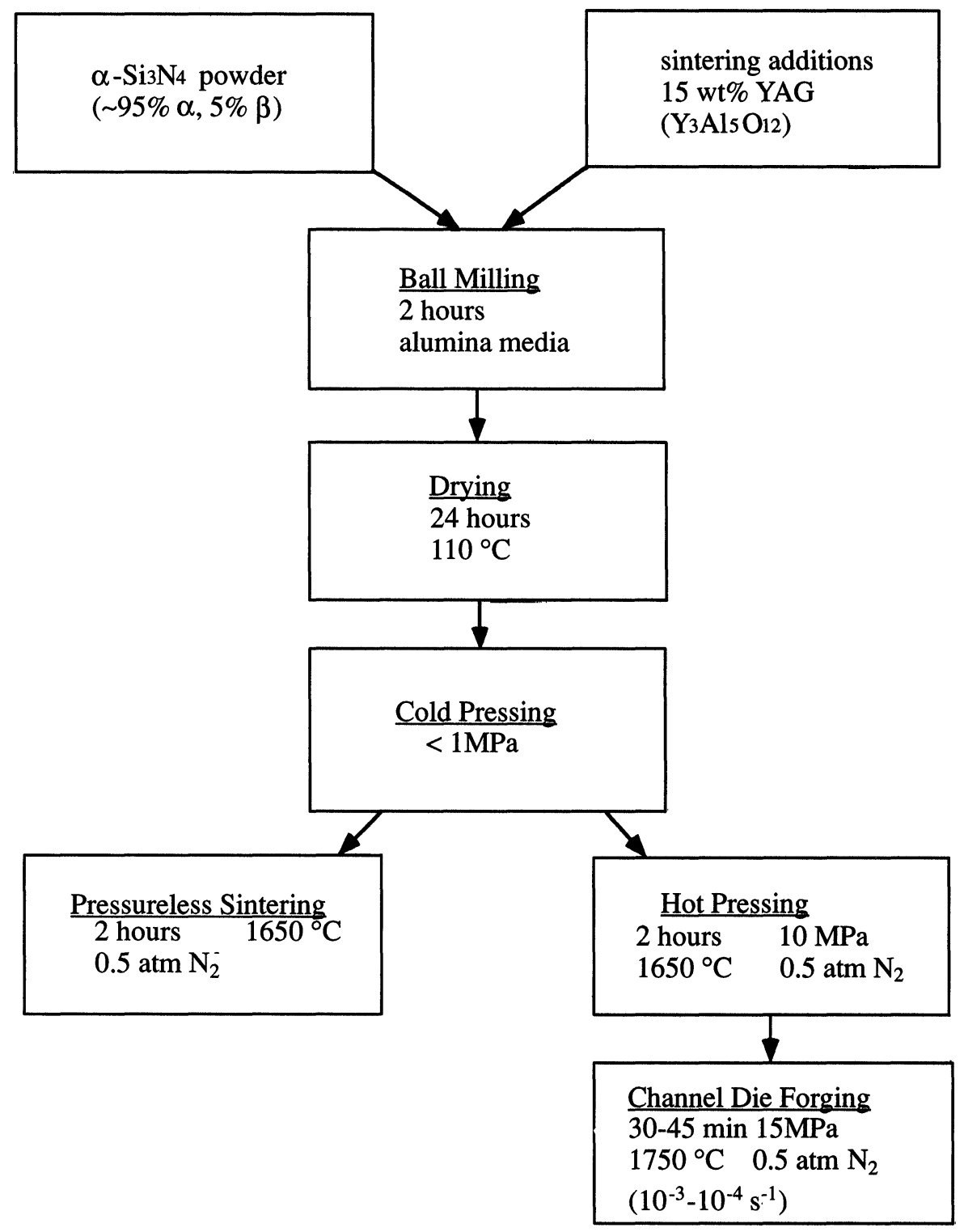

Figure 3 Flow chart of processing procedure.

Hot-pressed billets were cut to fit into a graphite channel die $1.3 \mathrm{~cm}$ in width and compressed to nominal height reductions of $30 \%, 40 \%, 50 \%, 55 \%$ and $70 \%$. An applied stress of $15 \mathrm{MPa}$ at $1750^{\circ} \mathrm{C}$ resulted in true strain rates between $10^{-3}$ to $10^{-4} \mathrm{~s}^{-1}$ in $0.5 \mathrm{~atm}$ of $\mathrm{N}_{2}$. The forging process took 30-45 minutes. A summary of the entire material and specimen preparation is schematically represented in Figure 3. 


\section{X-Ray Analysis}

The 002 basal pole figures for centered cross-section samples with various height reductions were measured directly using $\mathrm{Cu}-\mathrm{K} \alpha$ radiation and a pole figure goniometer with tilt angles $\chi=0^{\circ}$ to $80^{\circ}\left(5^{\circ}\right.$ increment), and rotation angles $\phi=0^{\circ}$ to $360^{\circ}\left(10^{\circ}\right.$ increment in continuous mode). The 200,101, 201 amd 301 incomplete pole figures were also measured for use in calculating the orientation distribution function (ODF) in the same specimens. Pole figure measurements were performed on the specimen face orthogonal to the extrusion direction, ED. Using popLA software, the ODF was calculated from harmonically completed pole figures by the WIMV (Williams-ImhofMatthies-Vinel) method (Kallend, 1991). To account for defocusing error, all pole figure measurements were corrected using a "random" sample prepared by pressureless sintering. Basal pole figures were then recalculated from the ODF.

To test repeatability in material production and pole figure measurements, plane sections from pole figures were taken for two separately channel-die-forged materials (plane strain condition) at 50\% height reduction. Figure 4 compares the degree of texture for 002 pole figures as a function of tilt angle toward the compression direction. The results indicate only a $2 \%$ difference in peak MRD (multiple of random distribution) in the normalized pole figures.

The 002 pole figure recalculated from the measured ODF corresponded within $\sim 2 \%$ of the directly measured (harmonically completed and normalized 002 only) pole figure at the $50 \%$ height reduction. Due to the high level of correlation between the recalculated and measured 002 pole figures, the latter type of pole figure is used in comparisons with modeling results at the other height reductions.

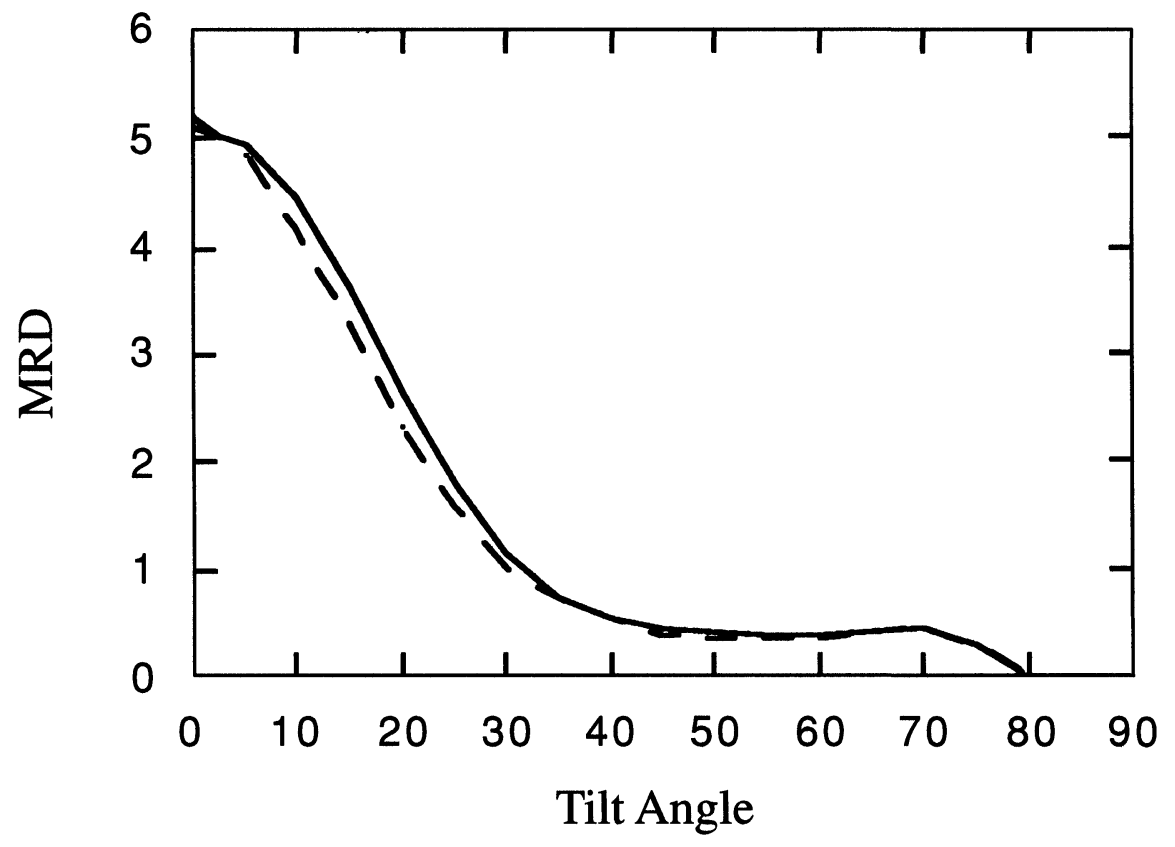

Figure 4 Plane section cuts of 002 pole figures along compression direction of separately processed $50 \%$ height reduction samples to demonstrate repeatability of process. 


\section{MODELING PROCEDURE}

Simulation of deformation processes was conducted using data generated by a FORTRAN program written by Lee (1994) for the plane strain compression condition. This program incorporated the Gay adaptation of the Jeffery model. An aspect ratio of 7.5 estimated from SEM micrographs was employed in the initial calculations.

The initial values for the simulation were supplied by a standard random-generator subroutine. For these simulations, the initial random orientations were kept constant to maintain consistency between simulations, i.e. the seeds for the number generators were kept constant. For this analysis a total of 4000 points was used per quadrant. The resulting values from the computer simulation were then converted into a readable format for the DIOR (DIscrete-ORientation) module of popLA (Kallend, 1991) (where each of the poles was assigned an equal weight. DIOR used a $5^{\circ}$ tilt and rotation bin range to create 002 pole figures. POPLA was used to complete the pole figure by symmetric operators on the modeled quadrant. All results were smoothed $5^{\circ}$ using a Gaussian filter in popLA.

\section{RESULTS AND DISCUSSION}

Peak intensities of experimentally determined 002 pole figures were compared with model results in Figure 5. Model predictions compared favorably for height reductions

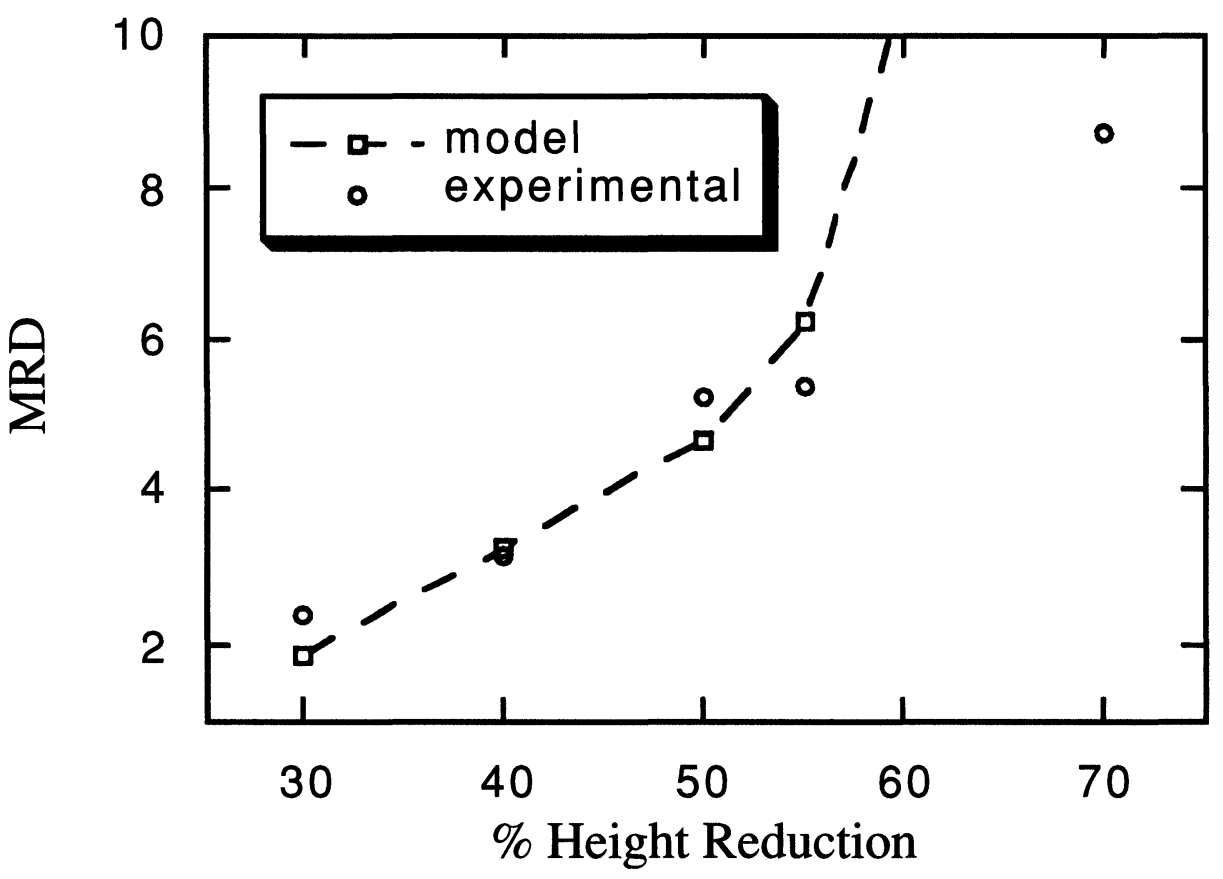

Figure 5 Comparison of model predictions to experimentally measured peak intensities. 


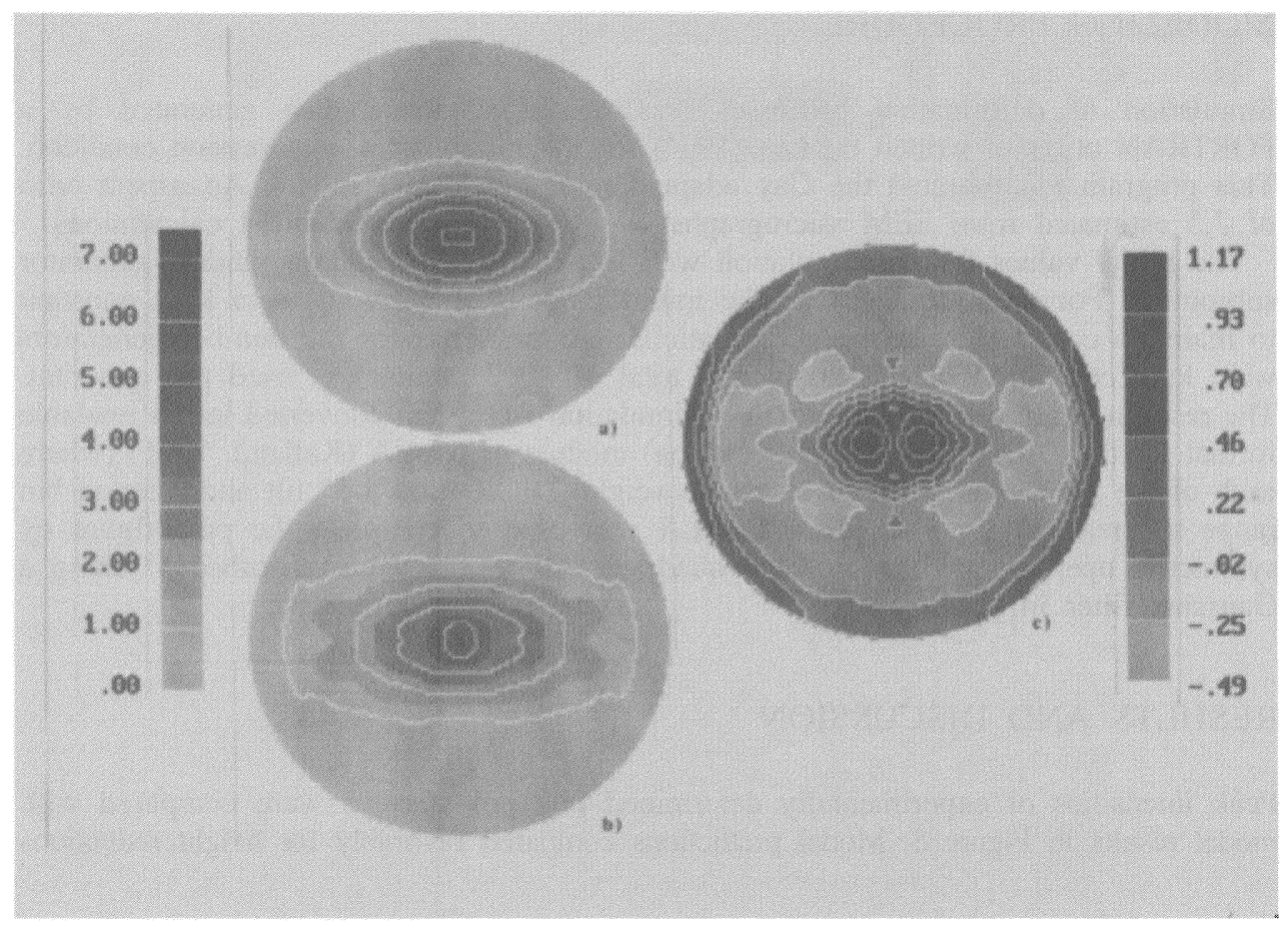

Figure 6(a) Experimental based and (b) model prediction 002 pole figures for 50\% height reduction. (c) Difference of experimental and model pole figures.

up to $50 \%$. To assess the performance of the model calculations at all orientations, the experimentally determined ODF and model predictions are compared in Figure 6a for a $50 \%$ height reduction. The shapes of the pole figures are very similar, indicating a flattening in the compression direction. Further comparison of the pole figures is made by taking the difference between the model predictions and the experimentally determined pole figures as shown in Figure $6 \mathrm{~b}$.

At a height reduction of $70 \%$ the model predicts a peak intensity of nearly 34 MRD, whereas the experimentally determined pole figure yields a peak of 8.7 MRD, Figure $7 \mathrm{a}$. The difference between model and experimental poles figures respectively is shown in Figure 7b, showing large deviation in both shape and intensity.

A possible rationalization for this deviation is due to die friction. Even so, strong friction was not readily apparent since the ends of the billets undergoing plane strain showed little curvature. Moreover, the X-ray samples were taken from the center of the billets, well away from die walls.

The most dominant term in this system, both mathematically and physically, is the aspect ratio. This can be seen in the governing equations for the Jeffery model. Thus any factor affecting this ratio will affect the results. Experimentally, factors such as fracture, welding, bundling and a distribution of aspect ratios would have a profound influence, creating a change in the effective ratio. This is exacerbated by the very large volume fractions of the rigid $\beta-\mathrm{Si}_{3} \mathrm{~N}_{4}$ phase. Additionally, stringent measurement of individual grain aspect ratio distributions in textured specimens is at best imprecise. 


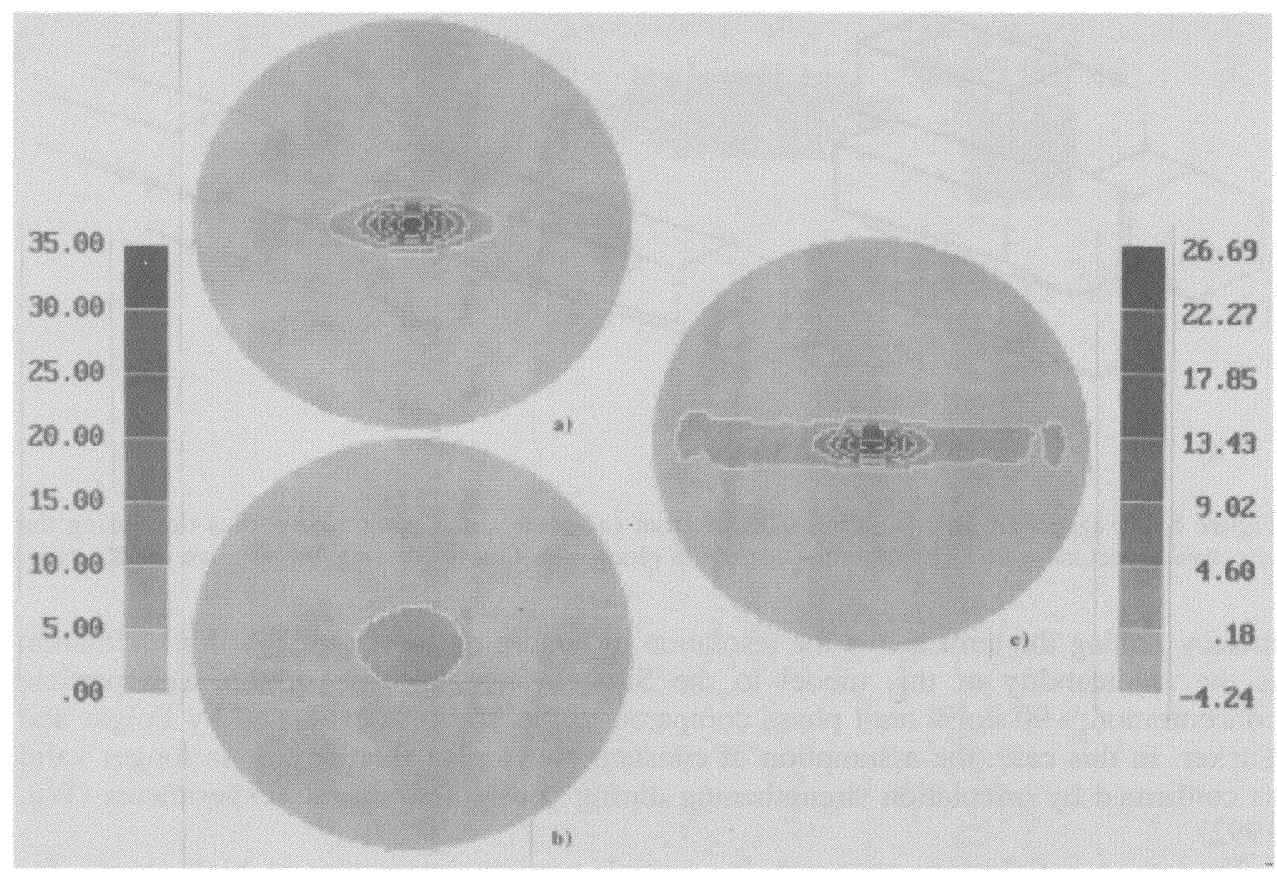

Figure 7(a) Experimental based and (b) model prediction 002 pole figures for $70 \%$ height reduction. (c) Difference of model and experimental pole figures.

The only influence found on the apparent aspect ratio from microstructural evaluations is the occurrence of regions containing highly oriented grains. These bundles of aligned grains, which may be welded radially, may effectively decrease $R$. No evidence of recrystallization or cavitation was found. $\mathrm{Wu}$ and $\mathrm{Chen}$ saw grain welding during superplastic elongation experiments with strain rates ranging from $3 \times 10^{-5}$ to $1 \times 10^{-3} \mathrm{~s}^{-1}$ in which interaction occurred by cavitation of the glassy phase and grain coalescence, resulting in an effective aspect ratio lengthening (Wu, 1992). Thus, the difference in applied stress for plane strain compression and superplastic tensile stretching may lead to differences in microstructural development that affect textures. Figure 8 depicts the types of grain welding anticipated in plane strain compression and superplastic tensile stretching. The hydrostatic compression of plane strain compression is expected to prevent the segmented welding observed by $\mathrm{Wu}$ and Chen (1992).

The question still remains whether or not interaction between grains can be accommodated by model parameters. The Folgar-Tucker "predictive" model incorporates the particle-particle interaction by superimposing an interaction term, a constant scalar of the strain tensor, to the Jeffery model. Essentially, the model is the sum of a hydrodynamic term, i.e. the Jeffery model, and an interaction term. One model shortcoming is that the interaction coefficient must be determined experimentally. The coefficient is not expressed as a function of the deformation so that any change in $R$ would alter the coefficient, including grain coalescence. A second limitation is the apparent negating impact the interaction term has on the Jeffery model. In the range suggested by experimental results, the coefficient (e.g. $~ 0.001$ ) effectively reduces the strain tensor, 


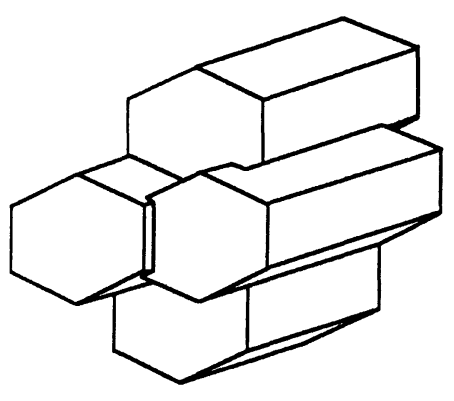

a)

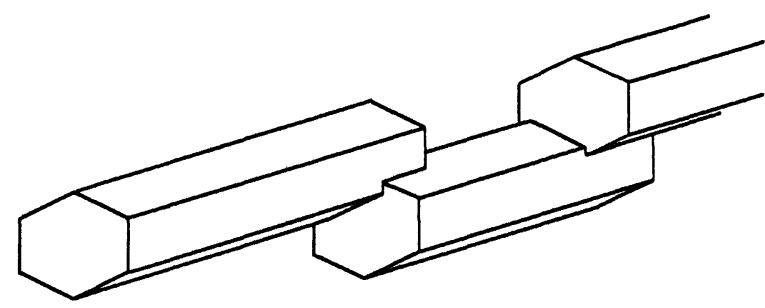

b)

Figure 8 Exaggerated grain welding induced from (a) plane strain compression thus decreasing the effective aspect ratio and (b) superplastic tensile elongation thus increasing the effctive aspect ratio.

thereby scaling the term below the resolution of texture measurements. A third limitation is the applicability of this model to the $\mathrm{Si}_{3} \mathrm{~N}_{4}$ system because of the high particle concentration, $\sim 90$ vol\% hard phase compared to the 16 vol\% evaluated by Folgar and Tucker. In this case, the assumption of constant Newtonian rheology is no longer valid as confirmed by orientation strengthening during constant strain-rate experiments $(\mathrm{Wu}$, 1992).

To date, a satisfactory approach for particle-particle interaction at high strains for concentrated solutions has not been found. A more fundamental theory of fiber orientation in concentrated suspensions would require an ability for prediction of terminal particle arrangements.

\section{SUMMARY}

Texture dependency of plane-strain compression forging of silicon nitride was measured for varying height reductions. Processing yielded directly measured peak intensities up to nine MRD for $70 \%$ height reduction. Modeling predictions compared favorably with experimentally determined pole figures of the alignment of 002 poles for plane strain height reductions up to $50 \%$. Divergence between model and experimental results at greater height reductions is attributable to physical interactions between grains. Currently, no model satisfactorily predicts the behavior at this and higher strains.

\section{Acknowledgements}

We would like to thank K. Kruger for his insightful discussions and assistance in this investigation. This research was supported by the National Science Foundation Grant No. 91-21948.

\section{References}

1. Advani, S. and Tucker, C. (1987). "The Use of Tensors to Describe and Predict Fiber Orientation in Short Fiber Composites." Journal of Rheology, 31, pp. 751-784.

2. Drew, R. A. L. (1988). "Silicon Nitride and SiAlON Ceramics - A Review", Canadian Metallurgical Quarterly, 27, pp. 59-64. 
3. Folgar, F. P. (1983). "Fiber Orientation Distributions in Concentrated Suspensions: A Predictive Model." Ph.D. Thesis, University of Illinois at Urbana-Champaign.

4. Folgar, F. P. and Tucker, C. L. (1984). "Orientation Behavior of Fibers in Concentrated Suspensions." Journal of Reinforced Plastic Composites, 3, pp. 98-119.

5. Gay, N. C. (1968). "The Motion of Rigid Particles Embedded in a Viscous Fluid During Pure Shear Deformation of the Fluid." Tectonophysics, 5, pp. 81-88.

6. Hosford, William F. (1993). The Mechanics of Crystals and Textured Polycrystals, Oxford, New York.

7. Jeffery, G. B. (1922). "The Motion of Ellipsoidal Particles Immersed in a Viscous Fluid." Proceedings of the Royal Society (London), A102, pp. 161-179.

8. Kallend, J. S., Kocks, U. F., Rollett, A. D. and Wenk, H.-R. (1991). "Operational Texture Analysis." Materials Science and Engineering, A132, pp. 1-11.

9. Lee, Farnjeng and Bowman, K. J. (1992). "Texture and Anisotropy in Silicon Nitride." Journal of the American Ceramic Society, 75, pp. 1748-1755.

10. Lee, Farnjeng and Bowman, K. J. (1994). "Texture Development via Grain Rotation in $\beta$-Silicon Nitride." Journal of the American Ceramic Society, 77, pp. 947-953.

11. Wu, Xin and Chen, I-Wei (1992). "Exaggerated Texture and Grain Growth in a Superplastic SiA1ON." Journal of the American Ceramic Society, 75, pp. 2733-2741. 